\title{
Detection of Acoustic Plasmons in Hole-Doped Lanthanum and Bismuth Cuprate Superconductors Using Resonant Inelastic X-Ray Scattering
}

\author{
Abhishek Nag®, ${ }^{1,}$ M. Zhu, ${ }^{2}$ Matías Bejas $\odot,{ }^{3}$ J. Li®,${ }^{1,4}$ H. C. Robarts, ${ }^{1,2}$ Hiroyuki Yamase $\odot,{ }^{5,6}$ A. N. Petsch, ${ }^{2}$ D. Song, ${ }^{7}$ \\ H. Eisaki, ${ }^{7}$ A. C. Walters, ${ }^{1}$ M. García-Fernández, ${ }^{1}$ Andrés Greco, ${ }^{3}$ S. M. Hayden ${ }^{2, \dagger},{ }^{2}$ and Ke-Jin Zhou ${ }^{1, \$}$ \\ ${ }^{1}$ Diamond Light Source, Harwell Campus, Didcot OX11 ODE, United Kingdom \\ ${ }^{2}$ H. H. Wills Physics Laboratory, University of Bristol, Bristol BS8 1TL, United Kingdom \\ ${ }^{3}$ Facultad de Ciencias Exactas, Ingeniería y Agrimensura and Instituto de Física de Rosario (UNR-CONICET), \\ Avenida Pellegrini 250, 2000 Rosario, Argentina \\ ${ }^{4}$ Beijing National Laboratory for Condensed Matter Physics, Institute of Physics, Chinese Academy of Sciences, Beijing 100190, China \\ ${ }^{5}$ International Center of Materials Nanoarchitectonics, National Institute for Materials Science, Tsukuba 305-0047, Japan \\ ${ }^{6}$ Department of Condensed Matter Physics, Graduate School of Science, Hokkaido University, Sapporo 060-0810, Japan \\ ${ }^{7}$ National Institute of Advanced Industrial Science and Technology (AIST), Tsukuba, Ibaraki 305-8560, Japan
}

(Received 1 August 2020; revised 18 October 2020; accepted 13 November 2020; published 14 December 2020)

High $T_{c}$ superconductors show a rich variety of phases associated with their charge degrees of freedom. Valence charges can give rise to charge ordering or acoustic plasmons in these layered cuprate superconductors. While charge ordering has been observed for both hole- and electron-doped cuprates, acoustic plasmons have only been found in electron-doped materials. Here, we use resonant inelastic x-ray scattering to observe the presence of acoustic plasmons in two families of hole-doped cuprate superconductors $\left(\mathrm{La}_{1.84} \mathrm{Sr}_{0.16} \mathrm{CuO}_{4}\right.$ and $\left.\mathrm{Bi}_{2} \mathrm{Sr}_{1.6} \mathrm{La}_{0.4} \mathrm{CuO}_{6+\delta}\right)$, crucially completing the picture. Interestingly, in contrast to the quasistatic charge ordering which manifests at both $\mathrm{Cu}$ and $\mathrm{O}$ sites, the observed acoustic plasmons are predominantly associated with the $\mathrm{O}$ sites, revealing a unique dichotomy in the behavior of valence charges in hole-doped cuprates.

DOI: 10.1103/PhysRevLett.125.257002

The electronic structure of high temperature superconducting layered cuprates [1] may be understood in terms of a hybridization between the $\mathrm{Cu} 3 d_{x^{2}-y^{2}}$ and $\mathrm{O} 2 p_{\sigma}$ orbitals, and a strong on-site Coulomb repulsion between electrons on the $\mathrm{Cu}$ sites [2-5]. When holes are introduced [see Fig. 1(a)], they reside preferentially in the so-called "charge-transfer band" (CTB) which is composed primarily of $\mathrm{O}$ orbitals [6]. In contrast, doped electrons enter the upper Hubbard band (UHB) and primarily reside on the $\mathrm{Cu}$ sites [5]. Despite this asymmetry in the electronic structure, charge order, a complex phase of periodically modulated charge-carrier density, has been observed ubiquitously on both the electron- and hole-doped sides of the phase diagram [7].

Surprisingly, a more widely observed mode of collective charge-density oscillation, the acoustic plasmon [8], has been rather elusive for the cuprates. In contrast to threedimensional (3D) metals, where long-range Coulomb interactions give rise to isotropic long-wavelength optical-like

Published by the American Physical Society under the terms of the Creative Commons Attribution 4.0 International license. Further distribution of this work must maintain attribution to the author(s) and the published article's title, journal citation, and DOI. gapped plasmons, out-of-phase oscillations of charges in neighboring planes of two-dimensional (2D) layered electron gases, form acoustic plasmons, whose energy tends to zero for small in-plane wave vectors [see Fig. 1(b)] $[9,10]$. Because of confinement of the doped charges to $\mathrm{CuO}_{2}$ planes and poor screening of out-of-plane Coulomb interactions by intervening dielectric blocks [see Fig. 1(c)], acoustic plasmons are also expected in the layered cuprates [11-13].

A remarkable discovery has been the recent observation of acoustic plasmons in electron-doped $\mathrm{La}_{2-x} \mathrm{Ce}_{x} \mathrm{CuO}_{4}$ (LCCO) and $\mathrm{Nd}_{2-x} \mathrm{Ce}_{x} \mathrm{CuO}_{4}[14,15]$ using $\mathrm{Cu} L_{3}$ resonant inelastic $\mathrm{x}$-ray scattering (RIXS). The excitations were found to have the strong out-of-plane dispersion expected for plasmons in layered systems. The situation in holedoped cuprates, however, has remained rather controversial. While $\mathrm{Cu} L_{3}$ RIXS did not detect plasmons in several families [16-18], $O K$ RIXS did detect excitations in $\mathrm{La}_{2-x}(\mathrm{Br}, \mathrm{Sr})_{x} \mathrm{CuO}_{4}$ that were interpreted as incoherent intraband transitions [19]. In zero-momentum optical investigation of $\mathrm{Bi}_{2} \mathrm{Sr}_{2} \mathrm{CaCu}_{2} \mathrm{O}_{8}$, apart from observation of optical plasmons at $1.1 \mathrm{eV}$, a low-energy non-Drude behavior was contemplated to be due to a band of acoustic plasmons [20]. However, electron energy-loss spectroscopy, a traditional probe for studying plasmons, observed only a high energy $(\sim 1 \mathrm{eV})$ overdamped optical plasmon 


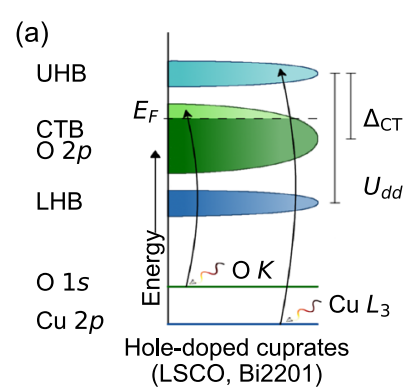

(b)

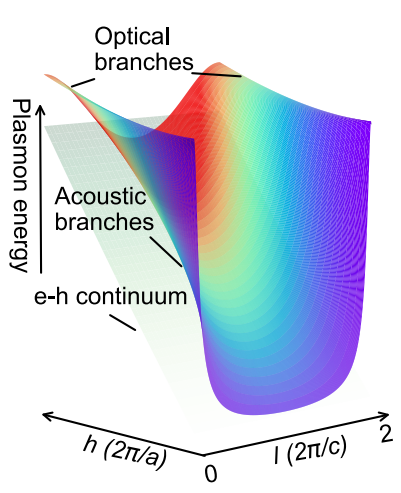

(c)

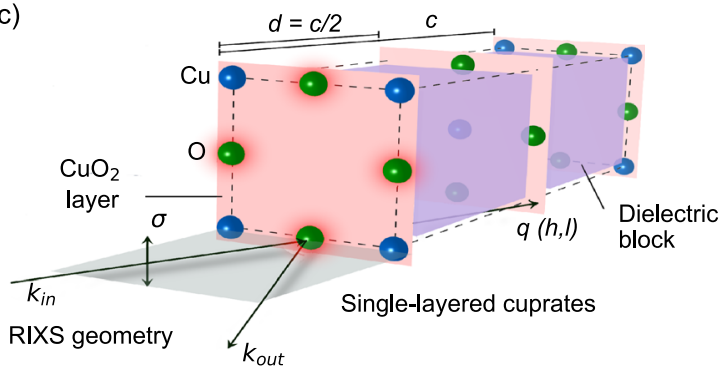

(e) $\quad \mathrm{Cu} L_{3}$-RIXS

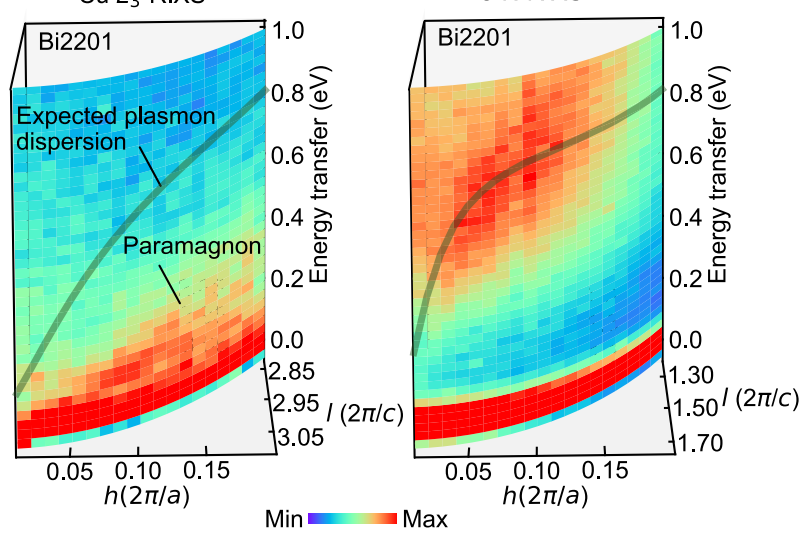

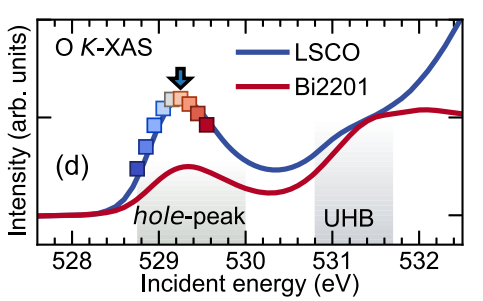

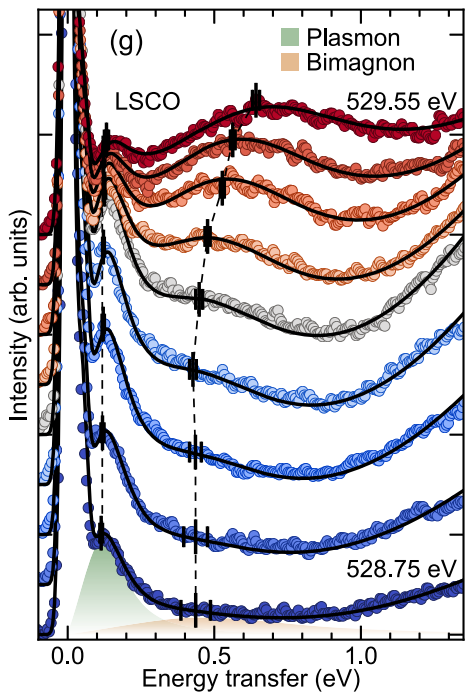

FIG. 1. (a) Schematic electronic structure of hole-doped cuprates in the Zaanen-Sawatzky-Allen scheme [2-5]. (b) Acoustic plasmon branches dispersing toward zero energy in the $h$ direction while maintaining a periodicity of $l=2$ in the $l$ direction for the layered structure shown in panel (c). (c) RIXS scattering geometry and a representative unit cell of single-layered cuprates. (d) Peak feature attributed to the doped-holes observed through the O $K$-XAS process [6]. The arrow shows the incident energy chosen to probe the plasmon dispersions in this work. RIXS intensity maps for Bi2201 at a fixed scattering angle of $114^{\circ}$ at (e) Cu $L_{3}$ edges and (f) $\mathrm{O} K$ edges. (g) Vertically stacked RIXS spectra for LSCO at $(h=0.03, l=1.00)$. Colors of the spectra correspond to incident energies shown in (d). Solid black lines are fits to the RIXS spectra. Vertical black bars are least-square-fit peak positions.

for small in-plane wave vectors in $\mathrm{Bi}_{2.1} \mathrm{Sr}_{1.9} \mathrm{CaCu}_{2} \mathrm{O}_{8+\delta}$ [21]. Acoustic plasmons in layered systems originate from the presence of conduction electrons and the long-range nature of the Coulomb interaction. Their absence in holedoped cuprates would conflict with our general understanding of the collective behavior of the doped charges.

In this Letter, we report that acoustic plasmons are indeed present in hole-doped cuprates from an extensive $\mathrm{O} \quad K$ RIXS study of $\mathrm{La}_{1.84} \mathrm{Sr}_{0.16} \mathrm{CuO}_{4}$ (LSCO) and $\mathrm{Bi}_{2} \mathrm{Sr}_{1.6} \mathrm{La}_{0.4} \mathrm{CuO}_{6+\delta}(\mathrm{Bi} 2201)$ over a wide range of inand out-of-plane momenta. The discovery of acoustic plasmons in the hole-doped systems remarkably illustrates the universal existence of low-energy collective excitations besides phonons and spin fluctuations across the cuprate phase diagram. Surprisingly, the observed acoustic plasmons are predominantly associated with the $\mathrm{O}$ sites in these hole-doped cuprates. Our results will therefore stimulate more studies of doped-hole charge dynamics, taking into account the three band model in the cuprates $[3,4]$.

Spectroscopically, $\mathrm{Cu} L_{3}$ RIXS and O $K$ RIXS directly probe the charge and magnetic excitations associated with the $\mathrm{Cu} 3 d$ and $\mathrm{O} 2 p$ orbitals, respectively, at the corresponding absorption peaks [Fig. 1(a)]. In order to compare the excitations associated with the two orbitals, highresolution RIXS spectra were collected at $\mathrm{Cu} L_{3}$ $(\Delta E \simeq 0.045 \mathrm{eV})$ and $\mathrm{O} K(\Delta E \simeq 0.043 \mathrm{eV})$ edges, at I21-RIXS beam line, Diamond Light Source, United Kingdom [22,23], in the scattering geometry shown in Fig. 1(c). All data presented here were obtained with incident $\sigma$ polarization (perpendicular to the scattering plane) to enhance the charge excitations [14]. Single crystals of LSCO and Bi2201 were cooled to their respective $T_{c} \mathrm{~s}$ of 38 and $34.5 \mathrm{~K}$, consistent with optimal hole doping of $p=0.16$, and $\mathrm{x}$-ray absorptions (XAS) were collected in total electron yield mode [see Fig. 1(d)] [23]. A survey was first made near the in-plane zone center with a fixed scattering angle on Bi2201 [Figs. 1(e) and 1(f)]. The lowenergy inelastic spectra at $\mathrm{Cu} L_{3}$ resonance are dominated by paramagnons without any noticeable signs of plasmons, similar to reports on other hole-doped systems [16-18]. At the $\mathrm{O} K$ edge hole-peak [6] however, a mode is found below $1 \mathrm{eV}$, dispersing toward the zero energy.

We next collected RIXS spectra by varying the incident energy $\left(E_{i}\right)$ across the hole peak in the O $K$ XAS of LSCO at $(h=0.03, l=1.00)$ [Fig. 1(d)] [6], as shown in Fig. 1(g). We denote momentum transfers along $h, k$, and $l$ directions 

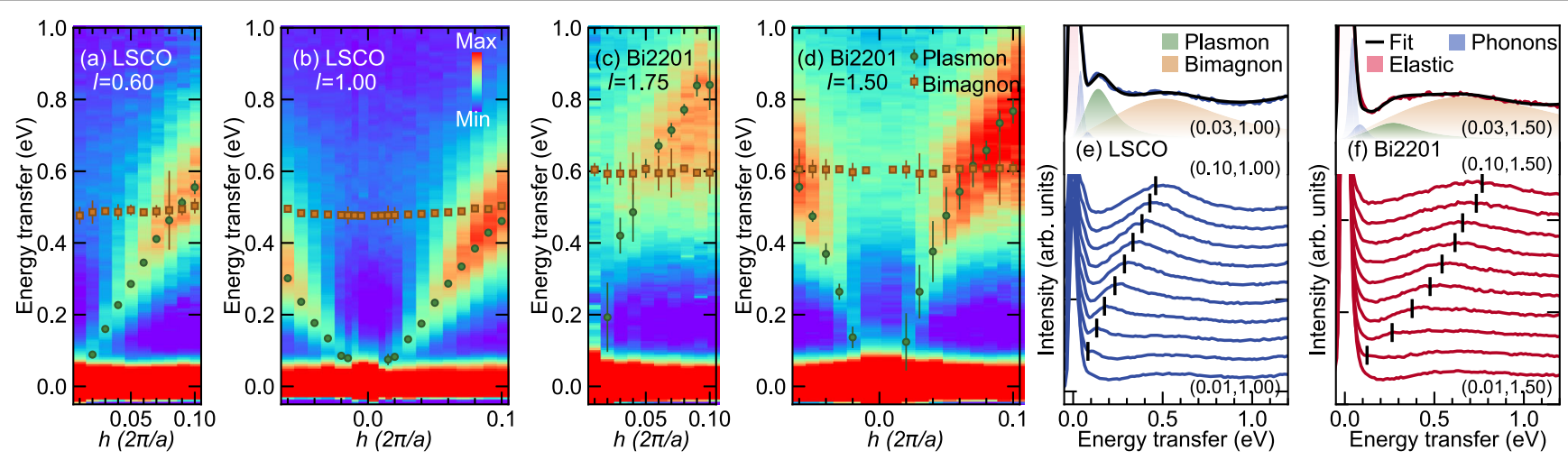

FIG. 2. RIXS intensity maps of LSCO for momentum transfer along the $h$ direction at (a) $l=0.60$ and (b) $l=1.00$ and of Bi2201 at (c) $l=1.75$ and (d) $l=1.50$. (e),(f) Top, representative RIXS spectra at mentioned $(h, l)$ values for LSCO and Bi2201. Shaded areas represent the different peak profiles [23]. (e),(f) Bottom, vertically stacked RIXS spectra from $h=0.01$ to 0.10 for LSCO and Bi2201 at $l=1.00$ and $l=1.50$, respectively.

in reciprocal lattice units, where $\mathbf{Q}=h a^{*}+k b^{*}+l c^{*}$ $\left(a^{*}=2 \pi / a, b^{*}=2 \pi / b, c^{*}=2 \pi / c\right), k=0$ if not stated explicitly. We find a broad feature at $\sim 0.5 \mathrm{eV}$ shifting toward higher energies with increasing $E_{i}$. With doping, the probability of scattering from doped charges in the intermediate state of RIXS increases [42]. Moreover, the energy shift of the magnetic excitations associated with incoherent charge excitations is enhanced in $\sigma$-polarized RIXS [43]. Thus, this feature can be ascribed to bimagnon excitations with an itinerant character [42]. We find an additional sharp mode, at $\sim 0.13 \mathrm{eV}$, whose energy remains constant with $E_{i}$. This is a signature of its coherent nature [15] and is in contrast with previous O $K$ RIXS results [19]. This feature cannot be due to two-particle electron-hole-like excitations, which are incoherent in nature. Neither can it be due to single magnons or paramagnons since $\Delta S=1$ spin-flip processes are forbidden at the $\mathrm{O} K$ edge [42]. To ascertain its origin, we explored further its dispersion in energymomentum space.

The broad feature seen in Fig. 1(g) is almost nondispersive in the $h$ direction, further confirming its assignment as bimagnons [42]. This can be seen from $(h, E)$ maps collected at constant $l$ values for LSCO and Bi2201 (Fig. 2). In contrast, the sharp mode disperses toward zero energy near the in-plane zone center in both systems. The RIXS spectra were fitted with a sum of Gaussians for the elastic line, phonons, and damped harmonic oscillator functions for the sharp mode and the broad feature [Figs. 2(e) and 2(f)] [23]. The dispersion and reduction of amplitude and width toward the in-plane zone center of this mode, is reminiscent of the acoustic plasmon behavior observed in electron-doped LCCO [14]. The spectral weight of this mode moves to lower energy as the doping is reduced (see Supplemental Material, Fig. S15 [23]). This is expected and consistent with the behavior of plasmons in LCCO [14,15]. However, the mode is strongly damped in comparison to LCCO (see Supplemental Material, Fig. S12 [23]), reflecting the stronger correlations (e.g., pseudogap) near optimal doping in hole-doped cuprates $[5,15]$.

The most stringent test for identifying the mode as plasmons in these systems is their $l$ dispersion. In the outof-plane direction, plasmons in layered electron systems have a periodicity of $2 \pi / d$ (where $d$ is the interlayer spacing), which corresponds to $l=2$ in these systems, with a minimum in energy at $l=1,3,5, \ldots$ [Fig. 1(c)]. LSCO and Bi2201 have interlayer spacings differing by a factor of $\sim 2$, allowing us to probe separate portions of this period. The sharp mode observed in Fig. 2, is found to disperse to a minimum energy value at $l=1$ for both systems. This can be seen in the $(l, E)$ maps collected at fixed $h$ values shown in Figs. 3(a)-3(d). This behavior fundamentally proves the presence of acoustic plasmons in hole-doped cuprates. We can exclude the previous interpretation of these excitations as incoherent intraband charge or electron-hole excitations which are 2D [19], without significant $l$ dependence [12]. We note that a limited out-of-plane dispersion study has also been done recently on underdoped LSCO [44].

The cuprates are strongly correlated electron systems [1]. As such, it is interesting to compare our experimental results with the recently developed calculations of plasmons in the framework of a $t-J-V$ model [12], although generic plasmon behavior can also be described by random-phase-approximation calculations $[11,13]$. For discussing the nature and origin of the 3D charge excitations in LSCO and Bi2201 we employed the minimal layered $t-J-V$ model $[12,23,45]$ :

$$
\begin{aligned}
H= & -\sum_{i, j, \sigma} t_{i j} \tilde{c}_{i \sigma}^{\dagger} \tilde{c}_{j \sigma}+\sum_{\langle i, j\rangle} J_{i j}\left(\vec{S}_{i} \cdot \vec{S}_{j}-\frac{1}{4} n_{i} n_{j}\right) \\
& +\frac{1}{2} \sum_{i, j} V_{i j} n_{i} n_{j} .
\end{aligned}
$$

Here, $t_{i j}$ represents the hopping parameter and $J_{i j}$ the exchange parameter. The $3 \mathrm{D}$ form of long-range 

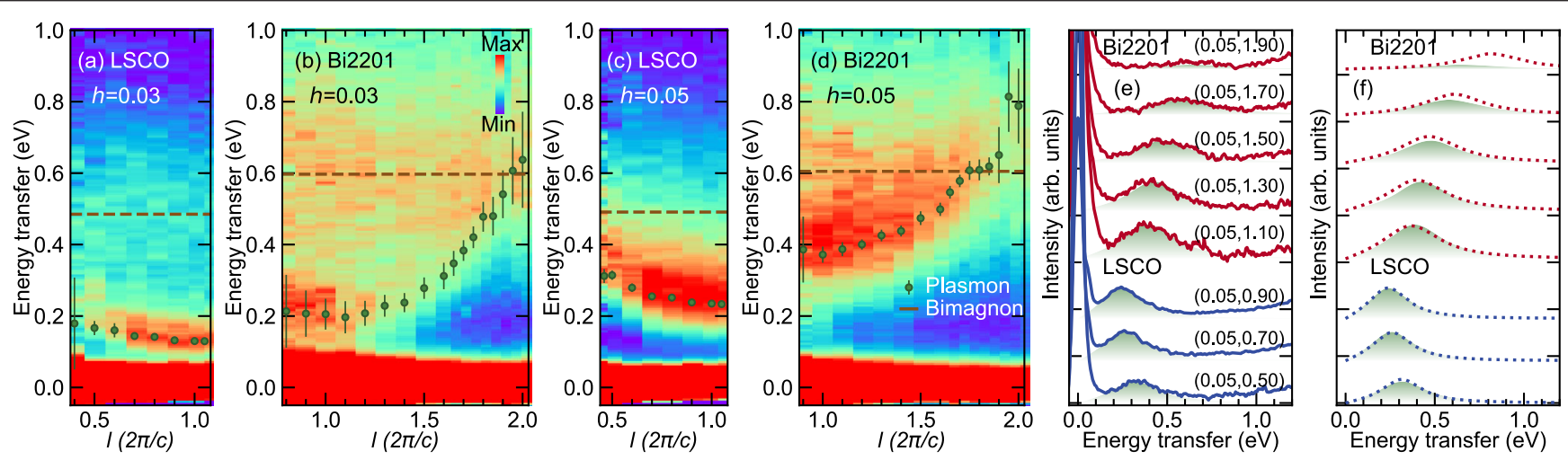

FIG. 3. RIXS intensity maps of LSCO and Bi2201 for momentum transfer along the $l$ direction at (a),(b) $h=0.03$ and (c),(d) $h=0.05$. (e) Representative RIXS spectra at mentioned $(h, l)$ values for LSCO (blue lines) and Bi2201 (red lines). Fitted peak profiles of bimagnons have been subtracted from the RIXS spectra to show the evolution of the plasmon peak (f) $\chi_{c}^{\prime \prime}(\mathbf{Q}, \omega)$ calculated from $t$ - $J$ - $V$ model for corresponding $(h, l)$ values in (e) for LSCO (Bi2201) are shown by the dotted blue (red) lines. Shaded green areas are the plasmon peak profiles obtained from RIXS data fitting. Different broadening factors $(\Gamma=0.29 t$ for Bi2201 and $0.2 t$ for LSCO) were chosen to replicate the line shapes of the two materials.

Coulomb interaction $V_{i j}$ used in Eq. (1) in momentum space is [46]

$$
V(\mathbf{Q})=\frac{V_{c}}{A\left(q_{x}, q_{y}\right)-\cos q_{z}},
$$

where $V_{c}=e^{2} d\left(2 \epsilon_{\perp} a^{2}\right)^{-1}$ and $A\left(q_{x}, q_{y}\right)=\alpha\left(2-\cos q_{x}-\right.$ $\left.\cos q_{y}\right)+1$ with $\alpha=\epsilon_{\|} / \epsilon_{\perp} /(a / d)^{2}$, $e$ the elementary charge, and high frequency in- $\left(\epsilon_{\|}\right)$and out-of-plane $\left(\epsilon_{\perp}\right)$ dielectric constants.

The imaginary part of charge susceptibility $\chi_{c}^{\prime \prime}(\mathbf{Q}), \omega$, obtained from the model resembles well the spectral shape of the plasmons for both systems, as shown in Fig. 3(f) at different $(h, l)$ values. This demonstrates that the charge excitations in RIXS, although influenced by resonance and polarization effects, can fundamentally be related to the charge-density response function. At $l$ values close to 2, the much larger suppression of plasmons compared to theory could be due to their decay through the incoherent charge channels associated with bimagnons or through umklapp scattering [14]. Severe suppression of the charge excitations in this region forbids us from detecting the optical plasmon branch in Bi2201 (see Supplemental Material, Fig. S6) [23]. In Figs. 4(a) and 4(b) we show that both the $h$ and $l$ direction plasmon dispersions extracted by fitting the RIXS spectra for LSCO and Bi2201 are also represented well by the $t-J-V$ model optimized independently for each material. The higher acoustic plasmon velocities in Bi2201 than LSCO seen in Figs. 4(a) and 4(b), arise mainly due to the larger interlayer spacing, considering the two systems have similar carrier densities and Fermi velocities (see Supplemental Material, Sec. V) $[9,14,23]$. Nevertheless, the remarkable similarity of the plasmon dispersions in the two different families suggests their ubiquitous existence in hole-doped cuprates.
In order to shed light on the possible existence of plasmons at $\mathrm{Cu}$ sites, we use the model described above to calculate the expected plasmon energies for both systems along the $(h, l)$ paths corresponding to the data for fixed scattering geometry. Neither in Bi2201 [Fig. 1(e)] nor in LSCO (Supplemental Material, Fig. S2 [23]), there is any spectral weight evident in $\mathrm{Cu} L_{3}$ RIXS spectra that can be assigned to plasmons [16,17]. This contrasts with the strong plasmon signals observed at the $\mathrm{O}$ sites. The
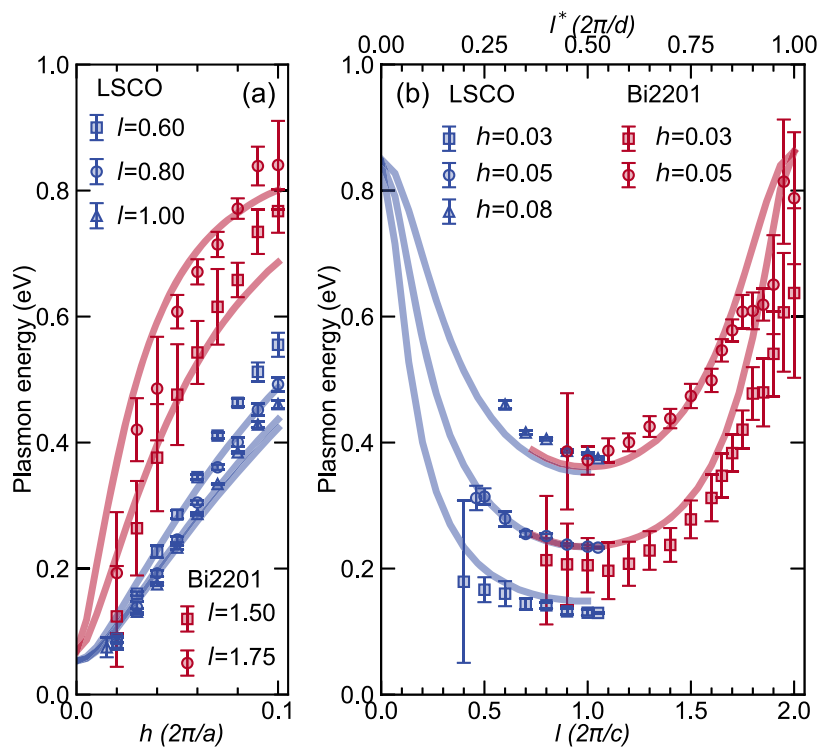

FIG. 4. (a) Plasmon energies in LSCO and Bi2201 for momentum transfer along the $h$ direction for different $l$ values and (b) for momentum transfer along the $l$ direction for different $h$ values, summarized from least-square fits of RIXS spectra. Continuous lines are plasmon dispersions obtained from $t-J-V$ model independently optimized for LSCO (blue) and Bi2201 (red). 
amplitude of plasmon is found to be highest close to $l=1$ for the O $K$ RIXS [Fig. 3 and Supplemental Material, Fig. S12(c) [23] ]. In the $\mathrm{Cu} L_{3}$ RIXS experiments close to the in-plane zone center, we probe near $l=3$ for Bi2201 and near $l=1.8$ for LSCO. Plasmons were, however, clearly observed in $\mathrm{Cu} L_{3}$ RIXS of electron-doped LCCO at similar $l$ values as in LSCO [14]. The nonobservance of plasmons at $\mathrm{Cu}$ sites in the present study of LSCO and Bi2201 could be therefore due to a combined effect of an $l$ dependence of plasmon spectral weight and a strong $\mathrm{O} 2 p$ character of the doped charges $[6,45,47]$. Further studies are required to clarify such site-dependent behavior, since in the Zhang-Rice singlet state scenario for hole-doped cuprates, a strong coupling is expected between the doped holes in the $\mathrm{O} 2 p_{\sigma}$ orbitals and the intrinsic holes of the $\mathrm{Cu} 3 d_{x^{2}-y^{2}}$ orbitals [45].

It is interesting to discuss our findings in the context of the charge order type of density modulation observed in hole-doped cuprates. When charge order is present, it has been observed using both $\mathrm{Cu} L_{3}$-edge and $\mathrm{O} K$-edge resonant X-ray scattering [48-50], both for short $(\sim 15 \AA)$ [50] and long ( 200 $\AA$ ) [48] correlation lengths. In common with other charge-density waves, the order has a valence charge modulation and associated atomic displacements [51], making it possible to be observed by nonresonant $\mathrm{x}$-ray scattering techniques [52]. Thus, it is likely that, in these systems, the charge ordering signal observed at the $\mathrm{Cu} L_{3}$ absorption peak is primarily due to atomic displacements caused by electron-phonon coupling, while the dominant signal at the $\mathrm{O} K$ hole peak reflects directly the valence charge modulation [49,53]. Because of the much higher frequencies of the dynamic plasmons, it may be that they couple weakly to the phonons, further reducing the possibility to observe any signature directly from the $\mathrm{Cu} 3 d$ orbitals.

The general existence of acoustic plasmons besides phonons and spin fluctuations in layered cuprates will lead to more investigations of charge dynamics in connection with the pseudogap phase, non-Fermi liquid behavior, and perhaps the superconductivity in cuprates [1]. Our results also suggest that the charge dynamics in hole-doped cuprates are mostly associated with the $\mathrm{O}$ sites, highlighting the importance of the three band model in the cuprates [3,4]. Going beyond, it would be interesting to utilize the site sensitivity of the RIXS technique to characterize plasmon behavior in other layered superconductors, like iron pnictides [54], having strong out-of-plane band dispersions, or the newly found nickelates in which $2 \mathrm{D} \mathrm{Ni} 3 d$ states strongly hybridize with $3 \mathrm{D}$ rare-earth $5 d$ states [55].

We thank W.-S. Lee, V. Kresin, J. Lorenzana, and S. Johnston for insightful discussions. All data were taken at the I21 RIXS beam line of Diamond Light Source (United Kingdom) using the RIXS spectrometer designed, built, and owned by Diamond Light Source. We acknowledge Diamond Light Source for providing the beam time on beam line I21 under Proposals No. SP20709 and No. MM24587. Work at Bristol was supported by EPSRC Grants No. EP/R011141/1 and No. EP/L015544/ 1. H. Y. was supported by JSPS KAKENHI Grants No. JP18K18744 and No. JP20H01856. We acknowledge T. Rice for the technical support throughout the beam times. We also thank G. B. G. Stenning and D. W. Nye for help on the Laue instrument in the Materials Characterisation Laboratory at the ISIS Neutron and Muon Source.

*abhishek.nag@diamond.ac.uk

†s.hayden@bristol.ac.uk

kejin.zhou@diamond.ac.uk

[1] B. Keimer, S. A. Kivelson, M. R. Norman, S. Uchida, and J. Zaanen, Nature (London) 518, 179 (2015).

[2] J. Zaanen, G. A. Sawatzky, and J. W. Allen, Phys. Rev. Lett. 55, 418 (1985).

[3] V. J. Emery, Phys. Rev. Lett. 58, 2794 (1987).

[4] C. M. Varma, S. Schmitt-Rink, and E. Abrahams, Solid State Commun. 62, 681 (1987).

[5] N. P. Armitage, P. Fournier, and R. L. Greene, Rev. Mod. Phys. 82, 2421 (2010).

[6] C. T. Chen, F. Sette, Y. Ma, M. S. Hybertsen, E. B. Stechel, W. M. C. Foulkes et al., Phys. Rev. Lett. 66, 104 (1991).

[7] R. Comin and A. Damascelli, Annu. Rev. Condens. Matter Phys. 7, 369 (2016).

[8] B. Diaconescu et al., Nature (London) 448, 57 (2007).

[9] A. L. Fetter, Ann. Phys. (N.Y.) 81, 367 (1973).

[10] A. L. Fetter, Ann. Phys. (N.Y.) 88, 1 (1974).

[11] V.Z. Kresin and H. Morawitz, Phys. Rev. B 37, 7854 (1988).

[12] A. Greco, H. Yamase, and M. Bejas, Commun. Phys. 2, 3 (2019).

[13] R. S. Markiewicz, M. Z. Hasan, and A. Bansil, Phys. Rev. B 77, 094518 (2008).

[14] M. Hepting et al., Nature (London) 563, 374 (2018).

[15] J. Lin et al., npj Quantum Mater. 5, 4 (2020).

[16] W. S. Lee et al., Nat. Phys. 10, 883 (2014).

[17] G. Dellea, M. Minola, A. Galdi, D. Di Castro, C. Aruta, N. B. Brookes et al., Phys. Rev. B 96, 115117 (2017).

[18] H. Miao et al., Proc. Natl. Acad. Sci. U.S.A. 114, 12430 (2017).

[19] K. Ishii, T. Tohyama, S. Asano, K. Sato, M. Fujita, S. Wakimoto et al., Phys. Rev. B 96, 115148 (2017).

[20] I. Bozovic, Phys. Rev. B 42, 1969 (1990).

[21] M. Mitrano et al., Proc. Natl. Acad. Sci. U.S.A. 115, 5392 (2018).

[22] https://www.diamond.ac.uk/Instruments/MagneticMaterials/I21.html.

[23] See Supplemental Material at http://link.aps.org/ supplemental/10.1103/PhysRevLett.125.257002 for sample information, RIXS experiments, additional RIXS data, description of the least-square data fitting and plasmon calculations using $t-J-V$ model, and includes Refs. [24-41]. 
[24] M. Minola, G. Dellea, H. Gretarsson, Y. Y. Peng, Y. Lu et al., Phys. Rev. Lett. 114, 217003 (2015).

[25] F. H. Vernay, M. J. P. Gingras, and T. P. Devereaux, Phys. Rev. B 75, 020403(R) (2007).

[26] A. Greco, H. Yamase, and M. Bejas, Phys. Rev. B 94, 075139 (2016).

[27] A. Foussats and A. Greco, Phys. Rev. B 70, 205123 (2004).

[28] O. K. Andersen, A. I. Liechtenstein, O. Jepsen, and F. J. Paulsen, Phys. Chem. Solids 56, 1573 (1995).

[29] P. Prelovšek and P. Horsch, Phys. Rev. B 60, R3735(R) (1999).

[30] T. Thio, T. R. Thurston, N. W. Preyer, P. J. Picone, M. A. Kastner, H. P. Jenssen, D. R. Gabbe, C. Y. Chen, R. J. Birgeneau, and A. Aharony, Phys. Rev. B 38, 905(R) (1988).

[31] M. S. Hybertsen, E. B. Stechel, M. Schluter, and D. R. Jennison, Phys. Rev. B 41, 11068 (1990).

[32] Y. Y. Peng et al., Nat. Phys. 13, 1201 (2017).

[33] P. A. Lee, N. Nagaosa, and X.-G. Wen, Rev. Mod. Phys. 78, 17 (2006).

[34] E. van Heumen, W. Meevasanam, A. B. Kuzmenko, H. Eisaki, and D. van der Marel, New J. Phys. 11, 055067 (2009).

[35] S. Uchida, T. Ido, H. Takagi, T. Arima, Y. Tokura, and S. Tajima, Phys. Rev. B 43, 7942 (1991).

[36] M. Suzuki, Phys. Rev. B 39, 2312 (1989).

[37] M. Hashimoto et al., Phys. Rev. B 77, 094516 (2008).

[38] M. Horio, K. Hauser, Y. Sassa, Z. Mingazheva, D. Sutter et al., Phys. Rev. Lett. 121, 077004 (2018).

[39] E. Pavarini, I. Dasgupta, T. Saha-Dasgupta, O. Jepsen, and O. K. Andersen, Phys. Rev. Lett. 87, 047003 (2001).
[40] G. Giuliani and G. Vignale, Quantum Theory of the Electron Liquid (Cambridge University Press, Cambridge, England, 2005).

[41] M. Turlakov and A. J. Leggett, Phys. Rev. B 67, 094517 (2003).

[42] V. Bisogni, M. Moretti Sala, A. Bendounan, N. B. Brookes, G. Ghiringhelli, and L. Braicovich, Phys. Rev. B 85, 214528 (2012).

[43] M. Minola, Y. Lu, Y. Y. Peng, G. Dellea, H. Gretarsson, M. W. Haverkort et al., Phys. Rev. Lett. 119, 097001 (2017).

[44] A. Singh et al., arXiv:2006.13424v1.

[45] F. C. Zhang and T. M. Rice, Phys. Rev. B 37, 3759(R) (1988).

[46] F. Becca, M. Tarquini, M. Grilli, and C. Di Castro, Phys. Rev. B 54, 12443 (1996).

[47] Y. Sakurai et al., Science 332, 698 (2011).

[48] P. Abbamonte, A. Rusydi, S. Smadici, G. D. Gu, G. A. Sawatzky, and D. L. Feng, Nat. Phys. 1, 155 (2005).

[49] A. J. Achkar, F. He, R. Sutarto, J. Geck, H. Zhang, Y.-J. Kim, and D. G. Hawthorn, Phys. Rev. Lett. 110, 017001 (2013).

[50] J. Li et al., Proc. Natl. Acad. Sci. U.S.A. 117, 16219 (2020).

[51] M. D. Johannes and I. I. Mazin, Phys. Rev. B 77, 165135 (2008).

[52] E. M. Forgan et al., Nat. Commun. 6, 10064 (2015).

[53] J. Fink et al., Phys. Rev. B 79, 100502(R) (2009).

[54] J. Paglione and R. L. Greene, Nat. Phys. 6, 645 (2010).

[55] M. Hepting et al., Nat. Mater. 19, 381 (2020). 